\title{
Training Japanese Speakers to Identify Nasal Codas of Mandarin Chinese
}

\author{
Ruining. Yang \\ Graduate School of Human and Environmental Studies, Kyoto University, Kyoto, Japan \\ Hiroaki. Nanjo \\ Academic Center for Computing and Media Studies, Kyoto University, Kyoto, Japan \\ Masatake. Danstuji \\ Academic Center for Computing and Media Studies, Kyoto University, Kyoto, Japan
}

\begin{abstract}
The aim of this study is to examine the effect of high variability training on "-n" and "-ng" in Mandarin Chinese. 46 Japanese native speakers who study Mandarin Chinese as a second foreign language at a university in Japan were divided into one experimental group and one control group. Participants assigned to the experimental group trained themselves using software, which was developed in our early research. Participants in the control group took Chinese lessons normally. Identification tasks (ITs) were conducted before and after the training. After 12 sessions, the average identification accuracy of nasal codas increased $7.1 \%(p<.05)$ in the experimental group, however, decreased $1.2 \%(p>.05)$ in the control group. Results of the training process prove that the training was effective for some participants, while had little effect on other participants. From the questionnaires, we found that there is a disagreement between the participants' subjective ability to identify nasal codas and the actual identification accuracy in ITs. Participants underestimate their ability to identify "-n" and "-ng" before training and overestimate their ability to identify "-n" and "-ng" after training. We made a formula to predict the accuracy of ITs (y) in the last 4 training sessions $(x): y=2.13 x^{2}-2.35 x+1.23$. This formula will help participants to grasp the training process and predict their identification ability.
\end{abstract}

Index Terms - Japanese speaker, nasal coda, Mandarin Chinese, training

\section{INTRODUCTION}

Second language (L2) learners usually face difficulties in distinguishing L2 contrastive phonetic sounds (Best \& Strange, 1992; Guion, Flege, Yamada \& Pruitt, 2000; Best, McRoberts \& Goodell, 2001), in the case that the L2 contrastive phonetic sounds are both categorized to a single first language (L1) phoneme (Aoyama, Flege, Guion, Yamada.T \& Yamada.R, 2004). In L2 learning, it is a significant issue for L2 learners to notice the differences between such phonetic sounds and distinguish them properly. This paper addresses how to train Japanese native speakers to be able to distinguish these kinds of $\mathrm{L} 2$ contrastive sounds.

There are studies that try to train Japanese English learners to identify such L2 sound pairs, specifically, English r and 1 (e.g., Lively, Pisoni, Akahane-Yamada, Thokura \& Yamada, 1994; Bradlow, Pisoni, Akahane-Yamada, Tohkura, 1997). Also, we can find studies about training a tonal contrast of Mandarin Chinese (e.g., Wang, Spence, Jongman \& Sereno, 1999; Wang, Jongman \& Sereno, 2003; Li, 2016), and pitch-accent patterns in Tokyo Japanese (e.g., Shport, 2016). They demonstrate that perceptual training could be effective in helping L2 learners to discriminate the confusing contrasts. In this paper, we deal with the training of L2 contrasts, with a particular focus on nasal codas in Mandarin Chinese.

In Mandarin Chinese, there are two types of nasal codas, "-n [n]" and "-ng [n]." Both nasals always appear after a single vowel or a diphthong in the coda position. The discrimination of these two nasal codas is very clear in Mandarin Chinese and the misuse of nasal codas sometimes brings about the confusion in understanding. For instance, suppose we pronounce the word "bú xìn" as "bú xìng," the meaning of the utterance would be "unhappiness" rather than "do not believe." The wrong pronunciation causes misunderstandings in communication. Thus, the discrimination of nasal codas is quite significant; however, less attention is paid to the contrast of "-n" and "-ng" in Mandarin Chinese.

A few studies have explored the issue of nasal codas from different perspectives. For example, Nozawa \& Cheon (2012) verified the identification of nasals in a coda position by native speakers of American English, Korean and Japanese. They compare Japanese speakers' identification accuracy of final syllable non-native nasals to that of English and Korean speakers. They report that Japanese speakers have lower identification accuracy, and they identified [y] most poorly; $[\mathrm{\eta}]$ is often mis-recognized as [n]. In Japanese, $[\mathrm{y}]$ is always an allophone of the phoneme /N/ and $[\mathrm{n}]$ is also an allophone of /N/ (Hatori, 1984, Hokama \& Sagawa, 1984); thus, Japanese speakers may recognize [n] as [n]. Aoyama (2003) reported that Japanese speakers had difficulty in discriminating [n] from [n] at the final position of 
syllable. The results of these study indicated that the L1 phonology of listeners strongly affects the identification of nasals in the coda position. Likewise, Japanese native speakers have difficulty in perceiving the nasal codas of Mandarin Chinese.

Ren (2006) reports perception experiment results for 50 Japanese speakers who learn Mandarin Chinese. The learners leaned to perceive "-ng" as “-n." Wang (2002) reports the relationship between perception and production with Mandarin Chinese nasal codas. In both the perception and production experiments, the identification accuracy of "-ng" exceeds that of "-n." In perception experiments, Japanese learners of Mandarin Chinese tended to perceive "-n" as "-ng." As just described, most of the previous research focused on the characteristics in the process of perceiving nasal codas and tried to investigate the error patterns of Japanese native speakers, and few research proposed an effective solution regarding the ability to discriminate between "-n" and "-ng" for Japanese native speakers. This is the first study to examine the effect of high variability phonetic training of Mandarin Chinese nasal codas "-n" and "-ng" for Japanese native speakers.

A high variability training paradigm was first introduced by Logan, Lively \& Pisoni (1991), which uses a variable sound stimulus consisting of a lot of phonetic environments with different talkers. Lively, Pisoni, Akahane-Yamada, Thokura \& Yamada.T (1993) extends the findings of Logan, Lively \& Pisoni (1991), and suggest that the high variability training paradigm encourages a long-term modification of listeners' phonetic perception. Training is not only limited to segmental features, but also suprasegmental features. For example, Wang, Spence, Jongman \& Sereno (1999) train American listeners to perceive Mandarin tones and show that the training leads to an improvement of $21 \%$, and it is effective. Shport (2016) trains native English listeners on Tokyo Japanese pitch-accent contrasts and verifies that the training leads to a greater improvement than mere exposure. These studies verify that a high variability training paradigm is effective in aiding L2 learners to discriminate between confusing contrasts. However, most of the previous studies just focus on the total improvement for the duration of the training, and pay little attention to the learning process of the participants. In this study, we investigate the training process of the participants and observe the characteristics during the training sessions.

Based on this background, in this paper, we investigate the issues as follows:

(1) Can Japanese speakers learning Mandarin Chinese improve their ability to perceive nasal codas "-n" and "-ng" via the high variability training, and how do they improve during the training process?

(2) Can an L2 learner understand his/her own identification ability of nasal codas "-n" and "-ng" appropriately?

(3) Is it possible to predict an L2 learner's identification ability at each training stage? If possible, we, as L2 learners, understand our own abilities and this should help us to recognize when we should finish the training.

There is no research that investigates the above three issues, and our research is novel in terms of above three points. We discuss the efficacy of the training to different learning levels of Mandarin Chinese, which is also original research.

In this paper, in section II, we describe the procedure and the preparatory work of the study. In section III, we introduce the experiments and results, giving the answers to the first research question. In section IV, we discuss the questionnaire and answer the second research question. In section $\mathrm{V}$, we make a formula to predict the ability of distinguishing nasal codas during the training and answer the third research question. Finally, we conclude in section VI.

\section{EXPERIMENTAL SETUP}

In this paper, we train Japanese native speakers so that they can discriminate the nasal codas "-n" and "-ng" of Mandarin Chinese. In this section, first of all we describe the procedure, and then, the Chinese utterances (stimuli) and training/testing software which we developed and used in the experiments are described. Also, the specification of participants in the training is described.

\section{A. Procedure}

We recruit Japanese native speakers as participants. First, they are asked to take part in the first identification task (IT-1) of "-n" and "-ng," and to answer a background questionnaire. Then, participants are divided into two groups: an experimental group and a control group. Participants assigned to the experimental group are asked to train themselves with our developed training software (Yang, Nanjo \& Dantsuji, 2017), which is described in section C. In the training period, they are required to work on the assignment 12 times. In this paper, we call everyday practice (assignment) a "session." Thus, the participants in the experimental group tackle 12 sessions in total. In every session, they first take a pre-test about "-n" and "-ng" identification, and then work on their discrimination abilities by listening to Chinese utterances (stimuli), and after the training, they take an identification test again (post-test). After the training period, participants assigned to the control group join the participants of the experimental group, and they take part in the second identification task (IT-2).

\section{B. Stimuli}

The stimuli used in the training are isolated utterances of one syllable (monosyllables) that could exist in Mandarin Chinese, all of which end with nasal codas. The reasons why we chose monosyllables as training stimuli are as follows. First, monosyllables easily cover real words and pseudo words. Second, monosyllables are not easily influenced by 
tones. If we use disyllables, we have to consider the influence of tones on the first syllable and the second syllable. Tones may influence the results of the identification tasks (ITs); in this study all the stimuli are pronounced in the first tone. Third, if real disyllable words are applied in the test, participants may infer the characters of the words and judge these disyllables from Pinyin. There are many possibilities of characters that are equivalent to monosyllables, but limited combinations of characters for disyllables.

The total number of sound stimuli is 173 . The stimuli were digitally recorded using a TASCAM HD-P2 recorder in a sound-proof room with a sampling rate of $44,100 \mathrm{~Hz}$. Three female native speakers and two male native speakers of Mandarin Chinese produced the stimuli (the three female native speakers are from Hebei Province, Shanxi Province and Heilongjiang Province, the two male native speakers are from Hebei Province and Beijing). All the producers of the stimuli are from areas in which nasal codas "-n" and "-ng" were discriminated clearly. We asked another 10 native speakers of Mandarin Chinese who are all from North China to judge "-n" and "-ng" for all the recorded stimuli, and we found that there was no problem with the stimuli.

The utterances of three native speakers (two females and a male) were selected as training stimuli and the utterances of the two remaining native speakers were used as stimuli of the ITs. In the ITs, 25 syllables were selected as test stimuli. Each stimulus was presented six times randomly; thus, the numbers of stimuli for the ITs is 150 .

\section{Training Software}

To conduct the ITs and training sessions, we used our training software (Yang, Nanjo \& Dantsuji, 2017). The training software runs on a Windows operating system as a GUI (Graphical User Interface) program. Users are instructed to listen to a Chinese syllable and to identify the final consonant in each utterance. There are two modes: a test mode and a practice mode. Both modes only allow users to click buttons, and the sounds in both modes can be replayed as many times as the users need. There was no problem with the usability of the training software (Yang, Nanjo \& Dantsuji, 2017). Unlike our previous training software setup (Yang, Nanjo \& Dantsuji, 2017), the number of utterances in the test mode is set to 20 to estimate the user's "-n/-ng" discrimination ability more effectively. Also, as for the difference to previous setup, we adopted three native speakers to increase a variability of the training stimuli.

There are 20 utterances in both the pre-test and the post-test and a two-alternative forced-choice IT is applied in the training session. Users might remember the order and give correct answers even if they cannot actually discriminate between "-n" and "-ng." Therefore, in the training software, the utterances in the pre-test and post-test are shown randomly.

The training software is also used for testing, that is, IT-1 and IT-2. In the ITs, the number of utterances is 150 (25 syllables $\times 6$ times).

\section{Participants}

We recruited 48 Japanese native speakers as participants. They are undergraduate students taking Chinese as a foreign language class at a public university in Japan: Kyoto University. They received rewards for their participation. The summary of the experiments is given in Table 1 . The number in the experimental group and the control group is 16 and 30, respectively. Eleven participants in the experimental group are beginner students who have been learning Mandarin Chinese for about only 1 to 2 months at IT-1, which is conducted before the training period. The other 5 participants are intermediate students who have been learning Mandarin Chinese for about 1 year at IT-1. The 30 participants in the control group are all beginners. At their Chinese class (beginners' class), the pronunciation and perception of Mandarin Chinese syllables is the main task for the first month. They have learned Pinyin-the Latin alphabet to show how to read a Chinese character- as a basis. All the participants received their knowledge of Mandarin Chinese from the same teacher; the instruction on nasal codas was conducted in a class.

The participants assigned to the experimental group are required to train themselves using the training software, which is installed on a CD-ROM. The participants are allowed to conduct their training at any place and any time. They are obliged to take 12 sessions during the training period (23rd May-7th June). The session duration is set to 15 minutes, which our software calculates and shows on a computer screen with the date of the training session. They are also obliged to send their training results to the author by email after each session. Specifically, screen shots of pre-test and post-test results, which our software automatically generates including the "-n/-ng" discrimination score and the date and time, are required to be submitted. 
TABLE 1.

SUMMARY OF THE EXPERIMENTS

\begin{tabular}{|c|c|c|c|}
\hline & & \multicolumn{2}{|l|}{ Training Experiments } \\
\hline & & Experimental Group & Control Group \\
\hline \multirow[t]{3}{*}{ Procedure } & Identification Task(IT-1) 150 stimuli & $\checkmark$ & $\checkmark$ \\
\hline & $\begin{array}{l}\text { Training } \\
\text { Each session } \\
>\quad \text { Pre-test }(20 \text { stimuli }) \\
>\quad \text { Practice }(15 \text { mins }) \\
>\quad \text { Post-test }(20 \text { stimuli })\end{array}$ & $\begin{array}{l} \\
12 \text { sessions } \\
\text { During } 23^{\text {rd }} \text { May to } 7^{\text {th }} \\
\text { June } 2016\end{array}$ & N/A \\
\hline & Identification Task(IT-2) 150 stimuli & $\checkmark$ & $\boldsymbol{V}$ \\
\hline \multicolumn{2}{|l|}{ Participants } & $\begin{array}{l}16 \text { ( } 11 \text { beginners and } 5 \\
\text { intermediates) }\end{array}$ & 30 (all beginners) \\
\hline
\end{tabular}

\section{EXPERIMENTS}

\section{A. Results of the Experimental Group and the Control Group}

For the experimental group, the identification accuracy of IT-1 was 57.3\%. After the 12 training sessions, the identification accuracy of IT-2 increased to $64.4 \%$. The paired sample $t$-test was used in these results and there was a significant difference between the identification accuracy. For the control group, the identification accuracy of IT-1 was $55.1 \%$, and the identification accuracy of IT-2 was $53.9 \%$. Contrary to increasing, the identification accuracy decreased a little. However, there was not a significant difference between the identification accuracy in the two ITs. The statistical results are given in Table 2.

TABLE 2.

THE STATISTICAL RESULTS OF THE EXPERIMENTAL GROUP AND CONTROL GROUP

\begin{tabular}{|l|l|l|l|l|l|}
\hline & Accuracy & $t$ & $d f$ & $p$ & Effect size \\
\hline Experimental group & $57.3-64.4 \%$ & 2.2498 & 15 & 0.0399 & 0.56245 \\
\hline Control group & $55.2-53.9 \%$ & -1.3907 & 29 & 0.1749 & \\
\hline
\end{tabular}

The high variability training paradigm in this study is effective for some Japanese speakers, while it seems to have a little effect on other participants. Twelve participants in the experimental group obtained an increase in the scores from IT-1 to IT-2. However, with regard to those participants whose identification accuracy ranged within $1 \%$, it is difficult to say whether they made progress or regressed. Due to limitations of space, some typical cases are given below. For example, participant $\mathrm{J}$ made a great improvement from $45.3 \%$ to $93.3 \%$. His training results are given in Fig.1. Rise and fall could be seen during the training period. In each training session, it was not necessary for participants to see an improvement after the training. Training results were analyzed every four sessions. The average accuracy increased gradually and remained stable from the sixth training onwards. When the standard deviation became smaller, the perceptual ability of "-n" and "-ng" also increased to a stable level.

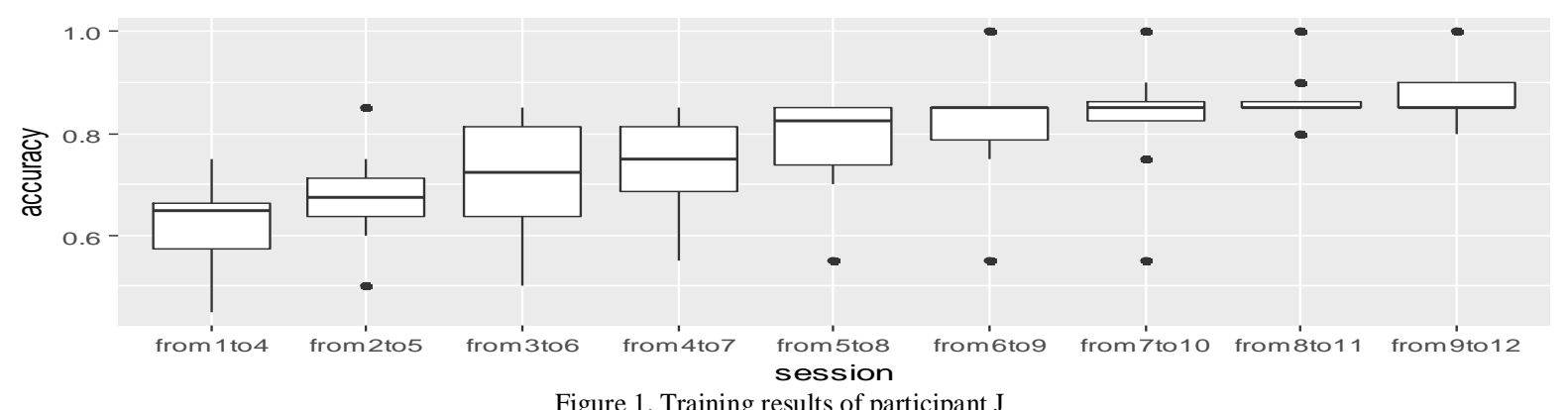

Boxplots: box region indicates the interquartile range; whiskers extend to extreme values; solid bar indicates the median; solid circle indicates the outlier.

However, from the training results of participant P (Fig. 2), we know it is not necessarily effective for every participant. The results of the post-tests did not show a great improvement, and the identification accuracy of 150 items in IT decreased from $58.7 \%$ to $54.0 \%$. 


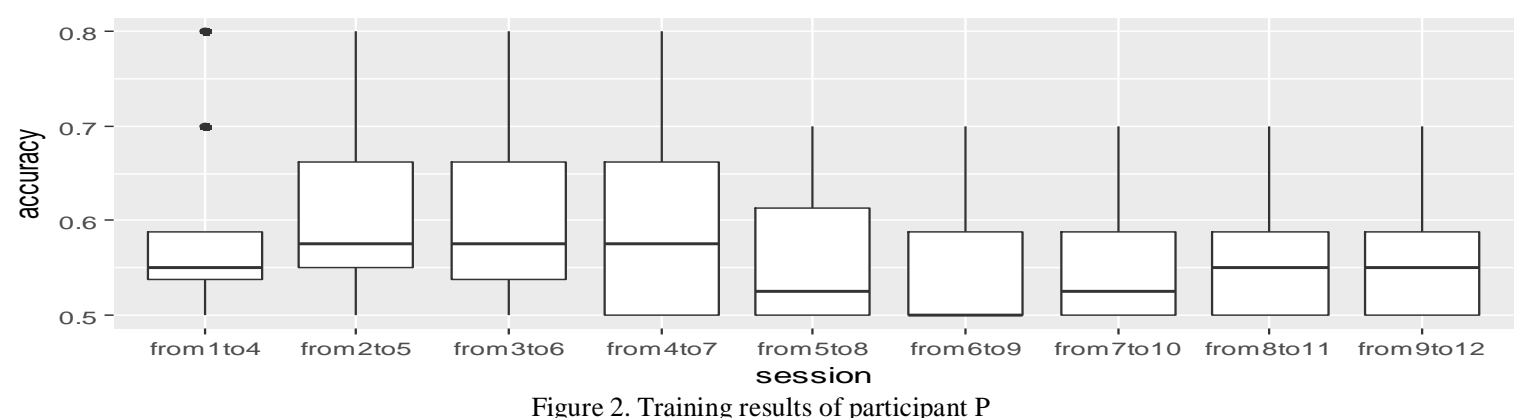

Boxplots: Conventions as in Figure 1.

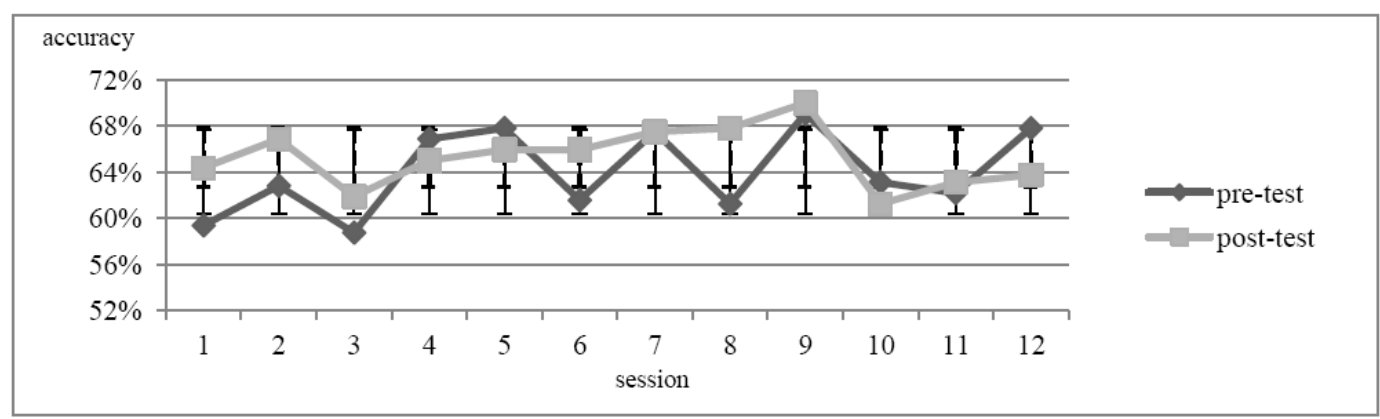

Figure 3. The average identification accuracy of training session Error bars indicate the standard error of the mean.

The average identification accuracy of all the participants in every training session is shown in Fig 3. Average identification accuracy increased from $59.4 \%$ to $63.8 \%$. During all the training sessions, the highest identification accuracy reached $70 \%$ after training. In the pre-tests, the identification accuracy was unsteady, rise and fall could be seen in almost every training session. We found that identification accuracy returned to the original level every few times. In Perrachione, Lee, Ha \&Wong (2011) and Iverson, Hazan \& Bannister (2005), although with some decrease occasionally, the results of training increased gradually. As regards our study, after 12 training sessions, a stable increasing curve has not been seen from the data. Training results may be related to the conditions of the participants, After the 12 training sessions, participants were still in the process of a slow beginning, there is no sign of steep acceleration. With the training period extended, participants may make more progress than before. However, it is difficult for participants to understand which stage they are in simply based on these unstable training results.

\section{B. Error Patterns}

To investigate if there is any error pattern in "-n" and "-ng," the identification accuracy of 150 items in IT were investigated respectively. Statistical results are shown in Fig.4. In the IT-1 of the experimental group, the identification accuracy of "-ng" was 58.9\%, and the identification accuracy of "-n" was 55.9\%. In the IT-2 of the experimental group, the identification accuracy of "-ng" was $66.2 \%$, and the identification accuracy of "-n" was $62.7 \%$. Moreover, in the IT-1 of the control group, the identification accuracy of "-ng" was $54.3 \%$, and the identification accuracy of "-n" was $55.9 \%$. In the IT-2 of the control group, the identification accuracy of "-ng" was $52.5 \%$, and the identification accuracy of "-n" was 55.3\%. No significant differences were observed between the results of "-n" and "-ng" in IT-1 and IT-2. It means learners have difficulties in perceiving both "-n" and "-ng" in Mandarin Chinese. This result is in accordance with the result of our previous study (Yang, Nanjo \& Danstuji, 2016). Wang (2002) states that Japanese learners of Mandarin Chinese tend to perceive "-n" to "-ng." However, this tendency is not seen in our research. In the experimental group, via the perception training of "-n" and "-ng," the identification accuracy of both kinds of nasal codas had increased respectively $(p<.001)$. In contrast, in the control group, the identification accuracy of "-n" and "-ng" remained stable. 


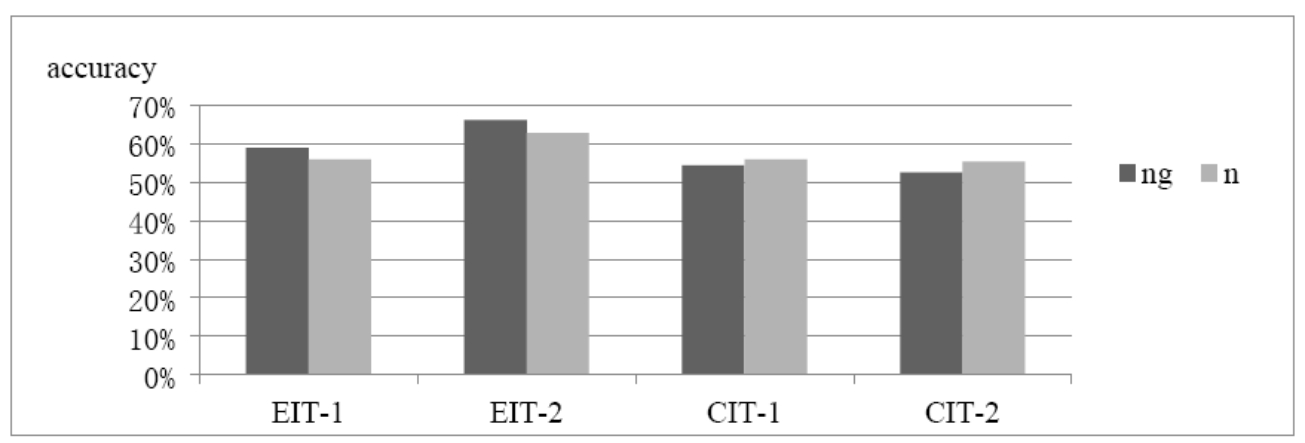

Figure4. Identification accuracy of "-n" and "-ng” in the experimental group and the control group EIT-1: accuracy of identification task before training of experimental group EIT-2: accuracy of identification task after training of experimental group CIT-1: accuracy of identification task before training of control group CIT-2: accuracy of identification task after training of control group

Via the results of the training and ITs, the answer to the first research question is very clear. "Can Japanese speakers learning Mandarin Chinese improve their ability to perceive nasal codas "-n" and "-ng" via the high variability training, and how do they improve via the training process?"

Compared with mere exposure, training is effective. However, observing the learning process and IT results of every participant, an increase was not obvious. Japanese learners of Mandarin Chinese can improve the ability to perceive the nasal codas "-n" and "-ng" via the high variability training. However, it is not effective for every participant. Some participants could make a great improvement through training, whereas some participants made no changes in identification accuracy during the training and ITs. For those learners for whom high variability training has little effect, another training paradigm should be considered. For example, more explanations could be given to learners before they start the training, or comparative example utterances should be included for learners to compare.

It is obvious that the Japanese learners of Mandarin Chinese could not distinguish these two nasal codas very well. However, after training with the software, the identification accuracy of experimental groups was upgraded to $64.4 \%$. This result provided some support that high variability training that keeps learners listening to the multiple words of L2 produced by different native speakers is effective in perceiving the confusing pairs. We surmise that the training period has had an effect on the training results; if the training continues, the identification accuracy may continue to increase.

\section{QUESTIONNAIRE}

After the training, all the participants in the experimental group were invited to answer a questionnaire about the nasal codas "-n" and "-ng." Questions were asked about the identification accuracy by subjective judgment. Participants were also welcome to make suggestions about the training software.

In the questionnaire, 93.8\% of the participants answered that they had learned the difference between "-n" and "-ng" in class. Participants were asked to judge how well they could distinguish the two pairs before and after the training, five choices were given from $0 \%$ (completely could not) to $100 \%$ (completely could). As shown in Fig.5, 43.8\% of the participants considered that they could not distinguish the two pairs completely before the training. $31.2 \%$ of the participants considered they could distinguish the two pairs at a rate of 25 percent, and the rest considered that they could distinguish at a rate of 50 percent. After the training, $43.8 \%$ of the participants considered they could distinguish the two pairs at a rate of 50 percent, and $37.5 \%$ of the participants considered that they could distinguish the two pairs at a rate of 75 percent. All the participants considered that they had made an improvement of perceiving "-n" and "-ng" through this training and $93.8 \%$ of the participants considered this training was useful. They were also asked what factors they used to distinguish "-n" and "-ng" (they could select as many choices as they used). 81.3\% of the participants considered that the length of the nasal codas was the distinguishing factor, and $43.8 \%$ of the participants used the length of the vowel to make a difference. It demonstrates that participants depend a lot on the factor of length to distinguish "-n" and "-ng."

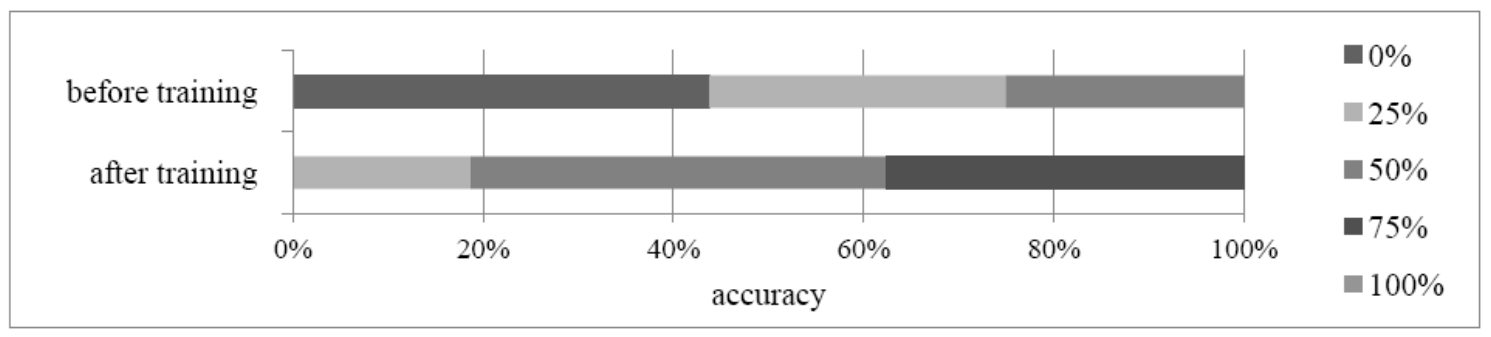

Figure5. Subjective identification accuracy before and after the training

Here we can answer the second research question "Can an L2 learner understand his/her own identification ability of 
nasal codas "-n" and "-ng" appropriately?" The answer is no. Participants underestimate their ability to identify "-n" and "-ng" before training and overestimate their ability to identify "-n" and "-ng" after training.

The identification accuracy judged by participants subjectively is at variance with the results of the ITs. Even though participants both in the experimental group and in the control group could discriminate nasal codas "-n" and "-ng" at a percentage of over $50 \%$, only few learners could judge their discriminating ability appropriately, and most participants considered they did worse than the actual identification accuracy. After the training, most participants considered that they could do better than before training. However, only $12.5 \%$ of the participants obtained a high identification accuracy of over $75 \%$. The results of the questionnaire demonstrate that Japanese learners of Mandarin Chinese have difficulty in understanding how well they could discriminate "-n" and "-ng." It is necessary to make learners recognize their current identification ability in order to train their perceptual ability effectively. We expect to predict the perceptual ability based on the results of the training so that participants can make full use of the training process.

\section{Discussion}

\section{A. Prediction of Discrimination Ability}

We made a regression analysis on the average accuracy of the last 4 training sessions and the accuracy of IT- 2 to verify their relationships. Based on the results of the 16 participants in the last 4 training sessions, the accuracy of IT- 2 is $60 \%$ related to the average accuracy of the last 4 training sessions. A polynomial approximation was made to predict the accuracy of IT after training from the accuracy of the last 4 training sessions. We designate the accuracy of the last 4 training sessions as $x$ and the accuracy of IT-2 as $y$. Participants' identification accuracy of IT-2 is equal to formula (1) with $\mathrm{R}^{2}=0.42$.

$$
y=1.98 x^{2}-2.15 x+1.17
$$

To verify the formula, we asked another 6 participants who have no learning experience in Mandarin Chinese to conduct the training in totally the same way with the participants in the experimental group. The predicted results and the actual results are shown in Table 3.

TABLE 3.

PREDICTED RESULT, ACTUAL RESULTS AND RESIDUALS

\begin{tabular}{|l|l|l|l|}
\hline Number & Actual result & Predicted result & Residual \\
\hline Predict1 & $49.3 \%$ & $59.5 \%$ & $10.2 \%$ \\
\hline Predict2 & $60.7 \%$ & $60.0 \%$ & $-0.7 \%$ \\
\hline Predict3 & $63.3 \%$ & $62.3 \%$ & $-1.0 \%$ \\
\hline Predict4 & $63.3 \%$ & $65.5 \%$ & $2.2 \%$ \\
\hline Predict5 & $64.0 \%$ & $61.4 \%$ & $-2.6 \%$ \\
\hline Predict6 & $72.7 \%$ & $75.4 \%$ & $2.7 \%$ \\
\hline
\end{tabular}

The data shows that the formula can be applied to predict the accuracy of IT after training based on the average accuracy of the last 4 training sessions. Except Predict 1, the accuracy of IT could be predicted comparatively correctly. To make the formula more accurate, we mixed the results of the 22 participants together and obtained a formula

$$
y=2.13 x^{2}-2.35 x+1.23
$$

with $\mathrm{R}^{2}=0.45$ (Fig.6). Participants can use this formula to understand their training process and predict the identification ability of nasal codas at any session. They can set a goal and train themselves with this training pattern.

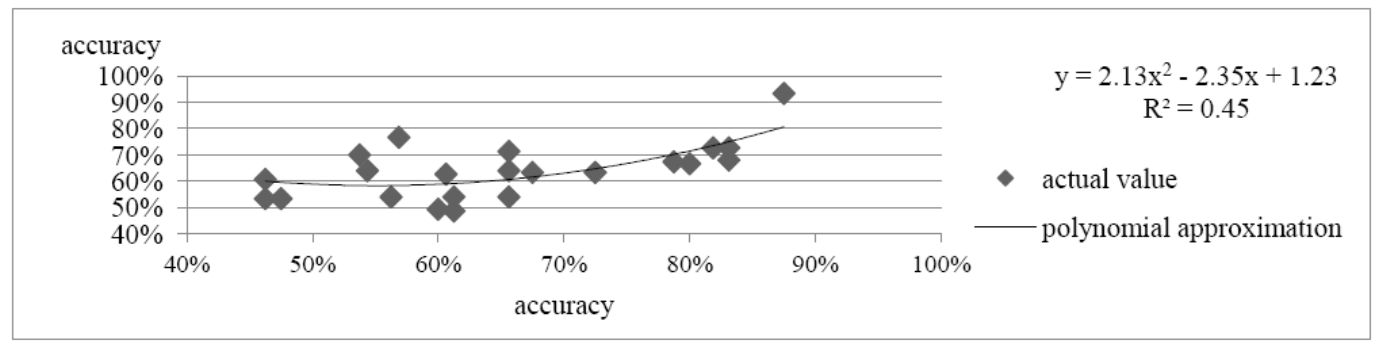

Figure 6. Perceptual predictiveness.

\section{B. The Effect of Training on the Different Learning Stages of Mandarin Chinese}

There were only two choices in the position of nasal codas, even though the participants knew nothing about Mandarin Chinese, they had a 50\% chance to give a right answer to the question. Consequently, identification accuracy less than 60\% was extremely low. Even though the identification accuracy among the intermediate level of Mandarin Chinese learners was higher than that of beginners of Mandarin Chinese, the identification accuracy did not reach $60 \%$. As the study progressed, learners did not make considerable improvements in perception (Yang, Nanjo \& Dantsuji, 2016). 
As mentioned above, there were 5 learners of intermediate level of Mandarin Chinese (Group A) and 11 beginners of Mandarin Chinese (Group B) in the experimental group. To verify the formula, we also asked 6 participants who had no learning experience in Mandarin Chinese (Group C) to conduct the training. The average identification accuracy of Group A in IT-1 was $61.9 \%$, whereas in IT-2 was $63.9 \%$. There was not a significant difference in the Wilcoxon signed-rank test between the two identification accuracy. However, in Group B, the average identification accuracy of IT-1 was $55.3 \%$, whereas in IT-2 was $64.6 \%$. There was a significant difference in the Wilcoxon signed-rank test. The average identification accuracy of IT-1 in Group C was $56.9 \%$, while in IT-2 was $62.2 \%(p>.05)$. Because of the limitation in the number of the participants, we could not obtain a significant difference to verify the influence that the learning period exerts on the training. However, participants in Group A whose learning period of Mandarin Chinese was the longest in the three groups demonstrated a weak increase after training and did not show an advantage from their learning experience. This problem should be included in future work. The statistical results are given in Table 4.

TABLE 4.

AVERAGE IDENTIFICATION ACCURACY OF DIFFERENT GROUPS

\begin{tabular}{|c|c|c|c|c|}
\hline & IT-1 & IT-2 & Difference & $p$ \\
\hline Group A & $61.9 \%$ & $63.9 \%$ & $2.0 \%$ & .19 \\
\hline Group B & $55.3 \%$ & $64.6 \%$ & $9.3 \%$ & $.02 *$ \\
\hline Group C & $56.9 \%$ & $62.2 \%$ & $5.3 \%$ & .21 \\
\hline
\end{tabular}

\section{CONCLUSION}

In this study, we trained Japanese learners of Mandarin Chinese to perceive nasal codas "-n" and "-ng" using training software. The average identification accuracy of nasal codas in the experimental group increased $7.1 \%(p=.0399<.05)$ after 12 training sessions; however, the average identification accuracy of nasal codas in the control group decreased $1.2 \%(p=.1749>.05)$. It is effective for some Japanese speakers who took part in the training and made improvements in perceiving both "-n" and "-ng." However, the identification accuracy remained the same as before training for some participants assigned to the experimental group. In view of the error patterns, Japanese learners have difficulties in perceiving both the nasal codas "-n" and "-ng" in Mandarin Chinese. We also demonstrated that participants with a longer learning period of Mandarin Chinese did not show an advantage over beginners in the training results. From the questionnaires, we found that there is a disagreement between the subjective judgement of the ability to identify nasal codas and the actual identification accuracy in the ITs. Participants underestimate their ability to identify "-n" and "-ng" before training and overestimate their ability to identify "-n" and "-ng" after training. As a result, we made a predicted formula to predict the accuracy of IT with the accuracy of the last 4 training sessions: $y=2.13 x^{2}-2.35 x+1.23$. This formula will help participants to understand the training process and predict their identification ability.

For the training paradigm, there are several places to improve. For example, more sounds of native speakers should be recorded and put into the program for listeners to get accustomed to the variations of the nasal codas. The influence of tones should be considered in the next experiments. Stimuli in disyllable should be included into the training to make the phonetic contexts changeable. According to the comments from the participants, it would be a good idea to explain how to distinguish "-n" and "-ng" in the program so that users can check the points while listening to the sounds. Moreover, the training period should be extended to verify whether a longer training period will lead to higher identification accuracy. Future work should be concentrated on these points.

\section{ACKNOWLEDGEMENTS}

The authors wish to thank the participants who took part in the experiments in this study.

\section{REFERENCES}

[1] Aoyama, K. (2003). Perception of syllable-initial and syllable-final nasals in English by Korean and Japanese speakers. Second Language Research 19.3, 251-265. doi:10.1191/0267658303sr222oa.

[2] Aoyama, K., J. E. Flege., S. G. Guion, R. Akahane-Yamada \& T. Yamada. (2004). Perceived phonetic dissimilarity and L2 speech learning: the case of Japanese /r/and English /1/ and /r/. Journal of Phonetic 23, 233-250. doi:10.1016/S0095-4470(03)00036-6.

[3] Best, C. T., G. W. McRoberts \& E. Goodell. (2001). Discrimination of non-native consonant contrasts varying in perceptual assimilation to the listener's native phonological system. Journal of the Acoustical Society of America 109, 775-794. doi:10.1121/1.1332378.

[4] Best, C. T. \& W. Strang. (1992). Effects of phonological and phonetic factors on cross-language perception of approximants. Journal of Phonetics 20.3, 305-330.

[5] Bradlow, A. R., D. B. Pisoni, R. Akahane-Yamada \& Y. Tohkura. (1997). Training Japanese listeners to identify English /r/ and /1/: IV. Some effects of perceptual learning on speech production. Journal of the Acoustical Society of America 101, 2299-2310.

[6] Guion, S. G., J. E. Flege, R. Akahane-Yamada \& J. C. Pruitt. (2000). An investigation of current models of second language speech perception: The case of Japanese adults' perception of English consonants. Journal of the Acoustical Society of America 107.5, 2711-2724. doi:10.1121/1.428657.

[7] Hatori, S. (1984). Onseigaku. Tokyo: Iwanami shoten.(in Japanese) 
[8] Hokama, S. \& M. Sagawa. (1984). Nihon gengogaku yōsetsu. Tokyo: Asakura shoten. (in Japanese)

[9] Iverson, P., V. Hazan \& K. Bannister. (2005). Phonetic training with acoustic cue manipulations: A comparison of methods for teaching English /r/-/l/ to Japanese adults. Journal of the Acoustical Society of America 118.5, 3267-3278. doi:10.1121/1.2062307.

[10] Lively, S. E., D. B. Pisoni, R. Akahane-Yamada, Y. Tohkura \& T. Yamada. (1994). Training Japanese listeners to identify English /r/ and /1/.III Long-term retention of new phonetic categories. Journal of the Acoustical Society of America 96, 2076-2087. doi:10.1121/1.410149.

[11] Li, Y. J. (2016). Effects of high variability phonetic training on monosyllabic and disyllabic Mandarin Chinese tones for L2 Chinese learners. Ph.D. dissertation, University of Kansas.

[12] Logan, J. S., S. E. Lively \& D. B. Pisoni. (1991). Training Japanese listeners to identify English /r/ and /1/: a first report. Journal of the Acoustical Society of America 89.2, 874-886. doi:10.1121/1.1894649.

[13] Nozawa, T. \& S.Y. Cheon. (2012). The identification of nasals in a coda position by native speakers of American English, Korean and Japanese. Journal of the Phonetic Society 16.2, 5-14.

[14] Perrachione, T. K., J. Lee, Louisa Y .Y. Ha \& Patrick C. M. Wong. (2011). Learning a novel phonological contrast depends on interactions between individual differences and training paradigm design. Journal of the Acoustical Society of America 130.1 , 461-472. doi:10.1121/1.3593366.

[15] Ren, X. (2006). nihonjin gakushūsha niyoru chūgokugo bion imbono chikaku oyobi seichō tono kanren. Journal of the Phonetic Society of Japan 10.7, 201-206. (in Japanese).

[16] Shport. I. A. (2016). Training English listeners to identify pitch-accent patterns in Tokyo Japanese. Studies in Second Language Acquisition 38, 739-769. doi:10.1017/S027226311500039X.

[17] Wang, Y., M. M. Spence, A. Jongman \& J. A. Sereno. (1999). Training American listeners to perceive Mandarin tones. Journal of the Acoustical Society of America 106, 3649-3658. doi:10.1121/1.428217.

[18] Wang, Y., A. Jongman \& J. A. Sereno. (2003). Acoustic and perceptual evaluation of Mandarin tone productions before and after perceptual training. Journal of the Acoustical Society of America 113.2, 1033-1043. doi:10.1121/1.1531176.

[19] Wang, Y. (2002). An experimental study on the perceptional and production of nasal codas by Japanese learners of Chinese Putonghua. Chinese teaching in the world 2, 47-60.

[20] Yang, R., H. Nanjo \& M. Danstuji. (2016). nihonjin gakushūsha niyoru chūgokugo bion imbono chikaku ni okeru tokuchō-senkō shiin ga haretsu shiin no baai-. Journal of the Japan Society for Speech Science 17,115-132. (in Japanese).

[21] Yang, R., H. Nanjo \& M. Danstuji. (2017). Development of ICT educational software for perceptional training of Mandarin Chinese nasal codas. INTED 2017 proceedings, 2664-2668. doi:10.21125/inted.2017.0732.

Ruining. Yang was born in Heilongjiang Province, China. She is currently a Ph.D. student at Kyoto University, Japan. She received her Bachelor Degree of Arts in Japanese from Harbin Institute of Technology in 2012 and Master Degree of Human and Environmental Studies from Kyoto University in 2015. Her research interests include Second Language Acquisition, phonetics, foreign language teaching and computer assisted language learning.

Hiroaki. Nanjo received the B.E. degree in 1999, the M.E. degree in 2001, and the Ph.D. degree in 2004 from Kyoto University, Kyoto, Japan. During 2004 to 2007, he was a Research Associate, and during 2007 to 2015, he was an Assistant Professor at Department of Media Informatics, Faculty of Science and Technology, Ryukoku University. From August 2015, he is an Associate Professor at Academic Center for Computing and Media Studies, Kyoto University. He has been working on speech recognition and understanding.

Masatake. Dantsuji received the B.S. and M.S. degrees of Letters from Kyoto University in 1979 and 1981, respectively. During 1990-1997, he stayed in Kansai University as associate professor. From 1997, he is a professor of Kyoto University. He has been working on phonetics, applied linguistics and computer assisted language learning. 